\section{Avaliação da tolerância à vancomicina em 395 cepas hospitalares de Staphylococcus aureus resistentes à oxacilina}

\author{
Evaluation of the tolerance to vancomycin in 395 oxacillin-resistant Staphylococcus aureus strains \\ isolated from Brazilian hospitals
}

Geraldo A. Oliveira ${ }^{1}$ Sabrina S. Okada ${ }^{2}$ Regiane S. Guenta ${ }^{2}$ Elsa M. Mamizuka ${ }^{3}$

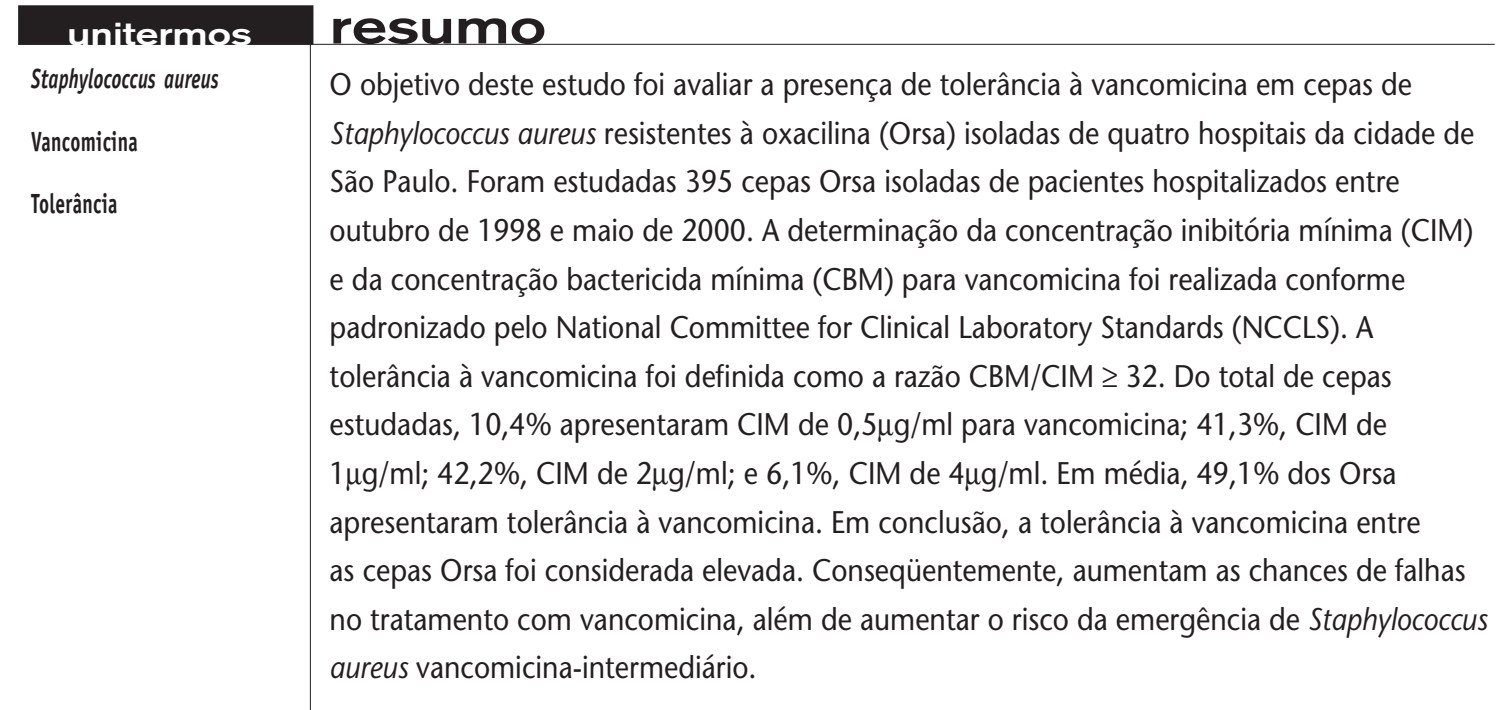

abstract

The objective of this study was to evaluate the presence of tolerance to vancomycin in oxacillinresistant Staphylococcus aureus (Orsa) strains isolated from four hospitals in the city of São Paulo.

From October/1998 to May/2000 we analysed 395 Orsa strains isolated from hospitalized patients. MIC and MBC to vancomycin were determined as standardised by National Committee

for Clinical Laboratory Standards (NCCLS). Tolerance was defined as the ratio MBC/MIC $\geq 32$. The results showed that $10.4 \%$ of the Orsa strains presented a MIC of $0.5 \mu \mathrm{g} / \mathrm{mL}$ for vancomycin, $41.3 \%$ presented a MIC of $1 \mu \mathrm{g} / \mathrm{mL}, 42.2 \%$ a MIC of $2 \mu \mathrm{g} / \mathrm{mL}$ and $6.1 \%$ a MIC of $4 \mu \mathrm{g} / \mathrm{mL}$. On average, $49,1 \%$ of the Orsa presented tolerance to vancomycin. We conclude that the tolerance

to vancomycin amongst the Orsa strains was considered high. These high levels of tolerance augment the chances of failure in the treatment with vancomycin, as well as enhancing the risk of emergence of vancomycin-intermediate Staphylococcus aureus. 
sadas por cepas Orsa $(5,17,23)$. Contudo, a emergência de Staphylococcus aureus com resistência intermediária à vancomicina (Visa) limitou consideravelmente as possibilidades de tratamento dessas infecções (12).

Apesar de o tratamento por tempo prolongado e o uso em larga escala da vancomicina serem considerados os principais fatores relacionados com a emergência de cepas Visa $(12,24,34)$, outros fatores correlacionados também poderiam favorecer 0 surgimento de resistência à vancomicina, como o desenvolvimento de tolerância, a concentração sérica da droga alcançada durante o tratamento e a capacidade de resposta imunológica do paciente, entre outros $(10,12,24,36)$. A tolerância aos antimicrobianos é definida como a capacidade de a bactéria mostrar-se sensivel à concentração inibitória mínima (CIM) do antibiótico, porém apresenta-se com maior capacidade de sobreviver na presença da droga, ou seja, não sofre a ação da concentração bactericida mínima (CBM) habitual $(2,10,15,36)$. Em termos práticos, a bactéria é incapaz de multiplicar-se, mas ainda pode permanecer viável no sítio da infecção. A tolerância foi inicialmente descrita em 1970 (37) e ocorre, principalmente, com antibióticos que agem na parede celular ( $\beta$-lactâmicos, cefalosporinas e glicopeptídeos) e bactérias gram-positivas $(2,10,15,36)$.

A tolerância à vancomicina em $S$. aureus foi relacionada com falhas terapêuticas em circunstâncias clínicas definidas, principalmente em septicemias, endocardites, osteomielites, meningites e em pacientes imunodeprimidos $(2,7,8,10,18,26,29,38)$. Tanto a tolerância como a resistência à vancomicina são consideradas fenômenos instáveis e induzidos, e aparecem durante o tratamento com a droga $(1,3$, $36,39)$. Conseqüentemente, uma vez tolerante à vancomicina, a bactéria poderia permanecer viável na presença da droga in vivo, permitindo o aumento da $\mathrm{CIM}$, já que teria tempo hábil para a adaptação à concentração do antimicrobiano $(22,37)$. Num próximo passo, poderia aumentar ainda mais os seus níveis de resistência e promover o seu crescimento no sítio da infecção, dependendo da concentração sérica do antimicrobiano. Portanto, a emergência de tolerância poderia ser considerada um dos primeiros passos no desenvolvimento de cepas Visa.

O objetivo desse estudo foi avaliar a freqüência do desenvolvimento de tolerância à vancomicina em cepas hospitalares de Staphylococcus aureus resistentes à oxacilina isoladas de quatro grandes hospitais da cidade de São Paulo.

\section{Materiais e métodos}

\section{Amostras bacterianas}

Foram estudadas 395 cepas Orsa provenientes de pacientes hospitalizados, isoladas no período compreendido entre outubro de 1998 e maio de 2000. As cepas foram isoladas de diversos materiais, como sangue, líquidos cavitários, queimaduras, abscessos contaminados, feridas cirúrgicas, lavado brônquico, ponta de cateter, secreções ortopédicas etc. Quatro hospitais públicos da cidade de São Paulo participaram desse estudo: Hospital das Clínicas da Faculdade de Medicina da Universidade de São Paulo (HCFMUSP), Hospital Emílio Ribas, Hospital Geral de Vila Penteado e Hospital Universitário da Universidade de São Paulo (USP).

Como a tolerância e a resistência à vancomicina em $S$. aureus são fenômenos instáveis e induzidos e as formas tolerantes e resistentes podem voltar ao comportamento regular da espécie quando armazenadas inadequadamente em laboratórios ou, ainda, podem desaparecer na ausência da pressão seletiva do antimicrobiano $(1,3,36,39)$, todas as amostras foram testadas imediatamente após o isolamento e a realização do antibiograma. Quando a determinação da CIM e da CBM não foi possível dentro do prazo previsto, as cepas de $S$. aureus foram imediatamente conservadas em caldo BHI (brain heart infusion), adicionado de $15 \%$ a $20 \%$ de glicerol, e armazenadas em freezer a $-80^{\circ} \mathrm{C}(11)$. Todas as cepas foram identificadas por meio da coloração de Gram e das provas de catalase e coagulase (14).

\section{Determinação da CIM e da CBM para vancomicina}

A CIM e a CBM foram determinadas conforme padronizado pelo National Committee for Clinical Laboratory Standards (NCCLS), utilizando-se a técnica de macrodiluição em caldo Müeller-Hinton $(11,20)$. A suspensão bacteriana na escala 0,5 de MacFarland foi preparada a partir de uma cultura em placa de ágar $\mathrm{BHI} /$ sangue incubada por 18 a 24 horas. Desta cultura, três a cinco colônias foram inoculadas em caldo BHI e incubadas sob agitação, por três a cinco horas, a $35-37^{\circ} \mathrm{C}$. Em seguida, a vancomicina (Eli Lilly, India- 
nápolis, EUA) foi diluída nas concentrações de 1024 a $0,25 \mu \mathrm{g} / \mathrm{ml}$, em diluição seriada de razão 2 , e ajustada para $1 \mathrm{ml}$ de volume final por tubo. Após a adição da bactéria-teste na concentração final de $5 \times 10^{5} \mathrm{UFC/}$ $\mathrm{ml}$, os tubos foram incubados por 24 horas, a $35-37^{\circ} \mathrm{C}$. A CIM foi definida como a menor concentração da droga capaz de inibir o crescimento visível do microrganismo $(11,20)$.

Para a determinação da CBM, os tubos foram homogeneizados por agitação durante a determinação da CIM, nos tempos de 20, 22 e 24 horas, para evitar a aderência da bactéria acima do menisco do meio contendo o antimicrobiano. Após a leitura da $\mathrm{CIM}, 10 \mu \mathrm{l}$ de cada tubo sem crescimento bacteriano visível foram transferidos para a superfície de uma placa de ágar BHI/sangue. Imediatamente após a absorção da gota, o material transportado foi espalhado com a ajuda de uma alça bacteriológica estéril. A CBM foi considerada a menor concentração da droga capaz de reduzir o número de bactérias viáveis em 99,9\% após 48 horas de incubação a $35-37^{\circ} \mathrm{C}(11,20,35)$. Staphylococcus aureus ATCC 29213 foi utilizado como controle de qualidade na determinação da CIM e da CBM $(11,20,35)$.

\section{Determinação da tolerância à vancomicina}

Para se determinar a tolerância do Staphylococcus aureus à vancomicina foi empregado o cálculo da razão CBM/CIM. Valores $\geq 32$ definem a bactéria como tolerante a esta droga $(18,20,35)$.

Quando se avalia a tolerância por meio da razão CBM/CIM, vários fatores podem interferir na determinação da CBM, como a fase de crescimento do inóculo inicial, o meio de cultura, o pH do meio, o tempo de incubação, a homogeneização dos tubos, o transporte de antibiótico para as placas de subcultivo, a sobrevivência da bactéria na parede do tubo acima do menisco do meio e as condições de armazenamento da bactéria-teste $(11,20,25,35)$. Para diminuir estas variáveis, nós seguimos rigorosamente as normas recomendadas pelo $\operatorname{NCCLS}(11,20)$.

\section{Análise estatística}

Para a análise estatística, foram utilizados o teste qui-quadrado e o teste exato de Fisher com nível de significância menor que 0,05 $(p<0,05)$.

\section{Resultados}

\section{Determinação da CIM e da CBM}

Do total de 395 cepas Orsa analisadas, 41 (10,4\%) apresentaram CIM de 0,5 $\mu \mathrm{g} / \mathrm{ml}$ para vancomicina; 163 (41,3\%), CIM de $1 \mu \mathrm{g} / \mathrm{ml} ; 167$ (42,2\%), CIM de $2 \mu \mathrm{g} / \mathrm{ml}$; e $24(6,1 \%)$ apresentaram CIM de $4 \mu \mathrm{g} / \mathrm{ml}$. Na Tabela 1 estão apresentados os percentuais dos valores das concentrações inibitórias mínimas, variáveis de 0,5 a $4 \mu \mathrm{g} / \mathrm{ml}$, obtidos em cada hospital estudado, e as diferenças significativas entre as CIMs observadas em cada hospital $(p=0,008)$.

Na Tabela 2 estão apresentados os percentuais dos valores da concentração bactericida mínima obtidos empregando-se a técnica de macrodiluição em tubo para a determinação da CIM e a conseqüente realização da CBM, conforme padronizado pelo NCCLS. No Hospital Geral de Vila Penteado, 52,9\% das cepas Orsa

\section{Tabela 1}

\begin{tabular}{l|cccc}
\hline Hospital & CIM de 0,5 $\mu \mathrm{g} / \mathrm{ml}$ & CIM de $1 \mu \mathrm{g} / \mathrm{ml}$ & CIM de $2 \mu \mathrm{g} / \mathrm{ml}$ & CIM de $4 \mu \mathrm{g} / \mathrm{ml}$ \\
$\begin{array}{c}\text { HCFMUSP } \\
(\mathrm{n}=137)\end{array}$ & $12,4 \%$ & $38 \%$ & $42,3 \%$ & $7,3 \%$ \\
$\begin{array}{c}\text { Hospital Emílio Ribas } \\
(\mathrm{n}=48)\end{array}$ & $20,9 \%$ & $47,9 \%$ & $31,2 \%$ & 0 \\
$\begin{array}{c}\text { Hospital Geral de Vila } \\
\text { Penteado (n= 174) }\end{array}$ & $4,6 \%$ & $41,4 \%$ & $46 \%$ & $8 \%$ \\
$\begin{array}{c}\text { Hospital Universitário } \\
\text { da USP (n=36) }\end{array}$ & $16,7 \%$ & $44,5 \%$ & $38,8 \%$ & 0 \\
\hline Total $(\mathrm{n}=395)$ & $10,4 \%$ & $41,3 \%$ & $42,2 \%$ & $6,1 \%$ \\
\hline
\end{tabular}

Percentuais dos valores da concentração inibitória minima para vancomicina encontrados em cada hospital estudado 
estudadas mostraram CBM $\geq 64 \mu \mathrm{g} / \mathrm{ml}$; no HCFMUSP, 51\%; no Hospital Emílio Ribas, 24,9\%; e no Hospital Universitário da USP, 33,3\%. Em média, 41,1\% das cepas Orsa analisadas apresentaram CBM $\geq 64 \mu \mathrm{g} / \mathrm{ml}$.

\section{Determinação da tolerância à vancomicina}

Entre as 395 cepas Orsa avaliadas para os valores da CIM e da CBM, 194 (49,1\%) demonstraram tolerância à vancomicina. Quando os resultados foram apresentados analisando-se cada hospital participante (Tabela 3), foram verificadas variações significativas nos percentuais de tolerância $(p=0,002)$. De maneira semelhante, o resultado comparativo do agrupamento dos dois hospitais com maiores e com menores taxas de tolerância apontou diferenças ainda mais expressivas ( $p=0,0002$ ). Por outro lado, quando comparamos as taxas de tolerância do HCFMUSP e do Hospital Geral de Vila Penteado, não encontramos diferenças significativas ( $p=0,4921)$. O mesmo aconteceu quando confrontamos os hospitais Emílio Ribas e Universitário da USP ( $p=0,7054)$.

Quando associamos CIM e tolerância, 12,2\% das cepas que apresentaram CIM de $0,5 \mu \mathrm{g} / \mathrm{ml}$ foram tolerantes à vancomicina. Já entre as cepas com CIM de $1 \mu \mathrm{g} / \mathrm{ml}$, o percentual aumentou para $22,7 \%$, subindo para $76,6 \%$ entre as cepas com CIM de $2 \mu \mathrm{g} / \mathrm{ml}$. Entre as cepas com CIM de $4 \mu \mathrm{g} / \mathrm{ml}$, o percentual foi de $100 \%$.

\section{Discussão e conclusões}

As falhas no tratamento de infecções causadas por S. aureus com vancomicina foram relacionadas a diversos fatores, como baixa atividade bactericida da droga, desnutrição e resposta imune reduzida do paciente, leucopenia e baixa concentração do antibiótico no sítio da infecção, entre outros $(12,24)$. No entanto, foi a emergência da resistência à vancomicina de $S$. aureus que culminou com a falência dessa opção terapêutica (12). Um fenômeno relacionado com a resistência é a tolerância $(4,22,36,37)$. Geralmente, a tolerância apresenta maior importância clínica em infecções onde a morte da bactéria depende diretamente da ação do antimicrobiano $(2,10,18,22,36)$.

Nesse estudo, 6,1\% das cepas Orsa estudadas apresentaram CIM de $4 \mu \mathrm{g} / \mathrm{ml}$ para a vancomicina. Porém, cepas Orsa expressando esta CIM foram encontradas somente no HCFMUSP e no Hospital Geral de Vila Penteado. Em adição, também se notou que estes hospitais apresentaram maiores percentuais de cepas com CIMs mais elevadas, quando comparados com o Emílio Ribas e o Universitário da USP (Tabela 1). Do mesmo modo, as maiores taxas de tolerância foram encontradas no Hospital Geral de Vila Penteado $(55,7 \%)$ e no HCFMUSP $(51,8 \%)$. Como a seleção de cepas in vivo com níveis mais elevados de resistência ocorre durante o tratamento com a droga $(1,3,12$, 36, 39), poderíamos pressupor, então, que os níveis mais elevados das CIMs seriam, provavelmente, decorrentes da maior prescrição de glicopeptídeos nestas instituições. Numa seqüência ordenada, o uso em larga escala de vancomicina estimula a emergência de tolerância à droga, e, finalmente, a elevada taxa de tolerância aumenta sensivelmente a probabilidade de falhas no tratamento com a vancomicina, além de favorecer a possível emergência de cepas Visa. Portanto, poderíamos inferir que o HCFMUSP e o Hospital Geral de Vila Penteado, assim como outros hospitais

Percentuais dos valores da concentração bactericida mínima obtidos nos

Tabela 2 hospitais estudados

\begin{tabular}{l|cccccccc}
\hline Hospital & $4 \mu \mathrm{g} / \mathrm{ml}$ & $8 \mu \mathrm{g} / \mathrm{ml}$ & $16 \mu \mathrm{g} / \mathrm{ml}$ & $32 \mu \mathrm{g} / \mathrm{ml}$ & $64 \mu \mathrm{g} / \mathrm{ml}$ & $128 \mu \mathrm{g} / \mathrm{ml}$ & $256 \mu \mathrm{g} / \mathrm{ml}$ & $512 \mu \mathrm{g} / \mathrm{ml}$ \\
$\begin{array}{c}\text { HCFMUSP } \\
(\mathrm{n}=137)\end{array}$ & $2,2 \%$ & $9,5 \%$ & $23,4 \%$ & $13,9 \%$ & $17,5 \%$ & $15,3 \%$ & $13,1 \%$ & $5,1 \%$ \\
$\begin{array}{c}\text { Hospital Emílio Ribas } \\
(\mathrm{n}=48)\end{array}$ & $8,3 \%$ & $25 \%$ & $31,3 \%$ & $10,4 \%$ & $6,2 \%$ & $10,4 \%$ & $8,3 \%$ & 0 \\
$\begin{array}{c}\text { Hospital Geral de Vila } \\
\text { Penteado ( } \mathrm{n}=174)\end{array}$ & $1,7 \%$ & $8 \%$ & $23,6 \%$ & $13,8 \%$ & $13,8 \%$ & $23 \%$ & $12,1 \%$ & $4 \%$ \\
$\begin{array}{c}\text { Hospital Universitário } \\
\text { da USP }(\mathrm{n}=36)\end{array}$ & $8,3 \%$ & $27,8 \%$ & $22,2 \%$ & $8,3 \%$ & $13,9 \%$ & $11,1 \%$ & $8,3 \%$ & 0 \\
\hline
\end{tabular}


com semelhante grau de complexidade de atendimento, poderiam apresentar maior risco de emergência de cepas Visa.

Apesar das elevadas taxas de tolerância (29\%-56\%) observadas em nossos resultados, os valores foram equiparáveis aos de outros estudos. Publicações anteriores mostraram que a presença de $S$. aureus tolerantes à vancomicina variou de $20 \%$ a 55\% (18, 21, 26, $27,29)$. Do mesmo modo, elevadas taxas de tolerância à vancomicina também podem ser observadas em outras espécies de bactérias gram-positivas (10, 40, 42). Por exemplo, cerca de $34 \%$ das cepas de Staphylococcus epidermidis foram tolerantes à vancomicina (40). Entre cepas de Streptococcus do grupo C e G, este percentual foi de 54\% (42).

Alguns fatores, além de interferir na determinação da tolerância no laboratório $(11,20,25,35)$, também podem favorecer a emergência da tolerância in vitro, como o pH reduzido do meio, a fase de crescimento do microrganismo e a concentração do microrganismo em estudo, entre outros $(11,18,20,25,35)$. Em circunstâncias semelhantes, estas alterações ambientais também podem ocorrer no sítio da infecção e causar a diminuição da atividade bactericida da droga in vivo, como: 1) o pH reduzido pode ser encontrado em fagossomas de polimorfonucleares, em líquido sinovial na artrite séptica, em líquido cefalorraquidiano nas meningites, em líquido pleural e intrabronquial nas pneumonias e em abscessos e empiemas; 2) o crescimento bacteriano lento pode ocorrer em osteomielites e dentro de formas vegetativas de endocardites; 3) a elevada concentração bacteriana e o crescimento na fase estacionária podem ser encontrados em endocardites, artrites sépticas e infecções profundas
(10). O efeito desses fatores sobre o desenvolvimento da tolerância in vivo não está completamente confirmado, mas suas influências negativas na evolução clínica do paciente durante o tratamento com vancomicina são bastante conhecidas (12).

Em média, 41,1\% das cepas Orsa estudadas apresentaram $C B M \geq 64 \mu \mathrm{g} / \mathrm{ml}$. Se a vancomicina atinge concentração sérica média nos picos de 20 a $50 \mu \mathrm{g} / \mathrm{ml}$ e nos vales de 5 a $15 \mu \mathrm{g} / \mathrm{ml}(30,41)$, conseqüentemente poderíamos deduzir que a tolerância com valores da $C B M \geq 64 \mu \mathrm{g} / \mathrm{ml}$ favoreceria a presença de bactérias viáveis no sítio da infecção, o que poderia se refletir em possíveis dificuldades terapêuticas, aumento da duração da antibioticoterapia e resultados insatisfatórios observados em pacientes críticos. No entanto, não se trata de uma afirmação absoluta, uma vez que existem vários fatores relacionados com faIhas terapêuticas, e esse poderia ser apenas um deles $(2,12,24)$.

Em nosso estudo, observou-se uma relação direta entre maior percentual de tolerância à vancomicina $\mathrm{e}$ níveis mais elevados da CIM. Houve um aumento paralelo dos valores, até que cepas com CIM de $4 \mu \mathrm{g} / \mathrm{ml}$ apresentaram 100\% de tolerância. Como o aumento do número de alvos para autolisinas (mureína) favorece a emergência de tolerância $(4,7,8,10,33)$ e como os níveis mais elevados da CIM, e até mesmo a resistência à vancomicina em S. aureus, são conseqüência do aumento da espessura da parede celular (maior síntese de ácido $\mathrm{N}$-acetilmurâmico e $\mathrm{N}$-acetilglicosamina - mureína) $(6,9,13,33)$, podemos afirmar, então, que a tolerância e a resistência estão diretamente relacionadas, e, portanto, a tolerância é um precursor da resistência (22). Teoricamente, a tolerân-
Percentual de cepas Orsa tolerantes à vancomicina em cada hospital estudado

\begin{tabular}{l|c}
\hline Hospital & Percentual de cepas Orsa tolerantes à vancomicina \\
\hline $\begin{array}{l}\text { Hospital Geral de Vila Penteado } \\
(\mathrm{n}=174)\end{array}$ & $55,7 \%$ \\
HCFMUSP & \\
$\quad(\mathrm{n}=137)$ & $51,8 \%$ \\
Hospital Universitário da USP & \\
$\quad(\mathrm{n}=36)$ & $33,3 \%$ \\
Hospital Emílio Ribas & \\
$(\mathrm{n}=48)$ & $29,2 \%$ \\
\hline
\end{tabular}


cia forneceria as condições básicas para que a bactéria pudesse permanecer viável por período prolongado no sítio da infecção, enquanto ocorre a exposição e a adaptação do microrganismo à concentração do antimicrobiano. Esta seria a situação requerida para induzir a expressão de níveis mais elevados da CIM in vivo (16). Ademais, confirmando esta teoria, Novak et al. (22) demonstraram que as cepas tolerantes à vancomicina são mais fáceis de ser transformadas em cepas resistentes in vitro que as cepas nãotolerantes.

Em S. aureus, a presença de tolerância à vancomicina ocorre principalmente entre cepas Orsa (10, 18). Este fato torna o tratamento de infecções causadas por este agente ainda mais difícil, uma vez que, no Brasil, as cepas Orsa são resistentes à maioria dos antimicrobianos com atividade antiestafilocócica (23). Como conseqüência, avaliar a tolerância à vancomicina em cepas Orsa isoladas de processos infecciosos, onde a atividade bactericida da droga é primordial para a eficácia do tratamento, pode ser fundamental para prognosticar o sucesso terapêutico.

Para o tratamento de cepas tolerantes, é necessário aumentar consideravelmente a dose e o tempo de ação do antimicrobiano, com os riscos inerentes. Estas exigências levantam dois pontos importantes no plano clínico: 1) o conhecimento da CBM na orientação da antibioticoterapia; 2) o aumento da concentração do antimicrobiano (no mínimo $32 \times \mathrm{CIM}$ ) no sítio da infecção $(7,8,31)$. Entretanto, estas alternativas nem sempre são disponíveis. $O$ aumento da concentração sérica do antimicrobiano poderá desenca- dear efeitos colaterais severos, e, ainda, o antibiótico poderá não alcançar a CBM desejada no sítio da infecção, prejudicando a eficácia do tratamento, principalmente em pacientes imunodeprimidos $(7,8,31)$. Além disso, a utilização de monoterapia com vancomicina em cepas tolerantes pode não ser segura, e a associação com outra droga bactericida é recomendada (19, $26,28,29,31$ ).

Em conclusão, a freqüência de cepas Orsa tolerantes à vancomicina foi considerada elevada nos hospitais estudados. A avaliação da tolerância aos glicopeptídeos é importante para prognosticar falhas no tratamento, e pode ser considerada um dos primeiros passos para prevenir e controlar a emergência de resistência à vancomicina em S. aureus. Enquanto protocolos e guias de monitorização da concentração sérica e da determinação da resistência à vancomicina não estão disponíveis, recomendamos a avaliação da tolerância e a pesquisa da resistência à vancomicina todas as vezes que a terapia antiestafilocócica não estiver respondendo apropriadamente. Maior vigilância dos níveis de sensibilidade à vancomicina deverá ser implantada, com vistas à prevenção da emergência de cepas Visa.

\section{Agradecimentos}

Agradecemos à dra. Adriana Dell'Aquila, do Hospital Geral de Vila Penteado, à dra. Flávia Rossi, do Hospital Emílio Ribas, e à dra. Evangelina Araújo, do HCFMUSP, que gentilmente forneceram as cepas Orsa para este estudo.

\section{Referências}

1. Aeschlimann, J.R.; Hershberger, E. \& Rybak, M.J. Analysis of vancomycin population susceptibility profiles, killing activity, and postantibiotic effect against vancomycin-intermediate Staphylococcus aureus. Antimicrob. Agents Chemother., 43: 1914-8, 1999.

2. Amsterdam, D. Susceptibility testing of antimicrobials in liquid media. In: Lorian, V. Antibiotics in laboratory medicine. 3.ed. Baltimore: Williams \& Wilkins, 1991. Vol. 3, p. 53-105.

3. Boyle-Vavra, S. et al. Reversion of the glycopeptide resistance phenotype in Staphylococcus aureus clinical isolates. Antimicrob. Agents Chemother., 44: 272-7, 2000.
4. Bryan, L.E. \& Godfrey, A.J. $\beta$-lactam antibiotics: mode of action and bacterial resistance. In: Lorian, V. Antibiotics in laboratory medicine. 3.ed., Baltimore: Williams \& Wilkins, 1991. Vol. 16, p. 599-664.

5. Chambers, H.F. Methicillin-resistance in Staphylococci: molecular and biochemical basis and clinical implications. Clin. Microbiol. Rev., 10: 781-91, 1997.

6. Cui, L. et al. Contribution of a thickened cell wall and its glutamine nonamidated component to the vancomycin resistance expressed by Staphylococus aureus Mu50. Antimicrob. Agents Chemother., 44: 2276-85, 2000. 
7. Freitas, C.C. et al. Tolerância a antibióticos em cepas de Staphylococcus aureus isoladas de pacientes do HUAP-UFF. Arq. Bras. Med., 65: 116S, 1991.

8. Freitas, C.C. O fenômeno da tolerância bacteriana aos antibióticos. J. Bras. Doenças Sex. Transm., 1: 103-8, 1989.

9. Hanaki, H. et al. Increase in glutamine-non-amidated muropeptides in the peptidoglican of vancomycinresistant Staphylococcus aureus strain Mu50. J. Antimicrob. Chemother., 42: 315-20, 1998.

10. Handwerger, S. \& Tomasz, A. Antibiotic tolerance among clinical isolates of bacteria. Rev. Infect. Dis., 7: 36886,1985

11. Hindler, J. Antimicrobial susceptibility testing. In: Isemberg, H.D. Clinical microbiology procedures handbook. Washington, D.C.: ASM Press, 1994. Vol. 1 , section 5 .

12. Hiramatsu, K. Vancomycin resistance in Staphylococci. Drug Resistance Updates, 1: 135-50, 1998.

13. Hiramatsu, K.; Ito, T. \& Hanaki, H. Mechanisms of methicillin and vancomycin resistance in Staphylococcus aureus. Ballière's Clin. Infect. Dis., 5: 221-42, 1999.

14. Kloos, W.E. \& Bannerman, T.L. Staphylococcus and Micrococcus. In: Murray, P.R. et al. Manual of clinical microbiology. 7.ed. Washington: American Society for Microbiology, 1999. Vol. 16, p. 264-82.

15. Koneman, E.W. et al. Diagnostic microbiology. 5.ed. New York: Lippincott, 1997. Vol. 15, p. 785-856.

16. Liu, H.H. \& Tomasz, A. Penicillin tolerance in multiply drug-resistant natural isolates of Streptococcus pneumoniae. J. Infect. Dis., 152: 365-72, 1985.

17. Lowy, F.D. Staphylococcus aureus infections. N. Engl. J. Med., 339: 520-32, 1998.

18. May, J.; Shannon, K. \& French, G. Glicopeptide tolerance in Staphylococcus aureus. J. Antimicrob. Chemother., 42: 189-97, 1998.

19. Musher, D.M. \& Fletcher, T. Tolerant Staphylococcus aureus causing vertebral osteomielitis. Arch. Intern. Med., 142: 632-4, 1982.

20. National Committee for Clinical Laboratory Standards (NCCLS). Methods for dilution antimicrobial susceptibility tests for bacteria that grow aerobically. Approved Standard M7-A5. 5.ed. Villanova, Pa., 2000. Vol. 20, n. 2.

21. Norden, C.W.; Keleti, E. Antibiotic tolerance in strains of Staphylococcus aureus. J. Antimicrob. Chemother., 7: 599-605, 1981.

22. Novak, R. et al. Emergence of vancomycin tolerance in Streptococcus pneumoniae. Nature, 399: 590-3, 1999.
23. Oliveira, G.A.; Levy, C.E. \& Mamizuka, E.M. Estudo do perfil de resistência de 626 cepas de Staphylococcus aureus isoladas de 25 hospitais brasileiros entre setembro de 1995 e junho de 1997. J. Bras. Pat., 36: 147-56, 2000.

24. Oliveira, G.A.; Levy, C.E. \& Mamizuka, E.M. Staphylococcus aureus apresentando resistência intermediária à vancomicina: mecanismos de resistência, detecção laboratorial e perspectivas de emergência no Brasil. J. Bras. Pat., 36(2): 96-102, 2000

25. Pelletier Jr., L.L. Lack of reproducibility of macrodilution MBCs for Staphylococcus aureus. Antimicrob. Agents Chemother., 26: 815-8, 1984.

26. Perry, J.D.; Jones, A.L. \& Gould, F.K. Glicopeptide tolerance in bacteria causing endocarditis. J. Antimicrob. Chemother., 44: 121-4, 1999.

27. Peterson, L.R. et al. Medium-dependent variation in bactericidal activity of antibiotics against susceptible Staphylococcus aureus. Antimicrobiol. Agents Chemother., 13: 665-8, 1978.

28. Rajashekaraiah, K.R. et al. Clinical significance of tolerant strains Staphylococcus aureus in patients with endocarditis. Ann. Intern. Med., 93: 796-800, 1980.

29. Reis, A.G.A.C. et al. Septicemia por Staphylococcus aureus em crianças: tolerância bacteriana à vancomicina $e$ poder bactericida do soro. Rev. Ass. Med. Brasil, 41: 47-52, 1995.

30. Reynolds, P.E. Structure, biochemistry and mode of action of glicopeptide antibiotics. Eur. J. Clin. Microbiol. Infect. Dis., 8: 943-50, 1989.

31. Sande, M.A. Antimicrobial therapy of infections in patients with Aids: an overview. J. Microb. Chemother., 23: 63-5, 1989.

32. Serazin, V.; Mougeot, C.; Libert, J.M. Staphylococcus aureus résistant à la méticilline et glycopeptides (vancomycine, teicoplanine): sélection de résistance in vitro. Path. Biol., 42: 323-7, 1994.

33. Sieradzki, K. \& Tomasz, A. Inhibition of cell wall turnover and autolysis by vancomycin in a highly vancomicyn-resistant mutant of Staphylococcus aureus. J. Bacteriol., 179: 2557-66, 1997.

34. Smith, T.L. et al. Emergence of vancomycin resistance in Staphylococcus aureus. N. Engl. J. Med., 340: 493 501, 1999

35. Stratton, C.W. In vitro testing: correlations between bacterial susceptibility, body fluid levels and effectiveness of antibacterial therapy. In: Lorian, V. Antibiotics in laboratory medicine. 3.ed. Baltimore: Williams \& Wilkins, 1991. Vol. 24, p. 849-79.

36. Tavares, W. Manual de antibióticos e quimioterápicos antiinfecciosos. 2.ed. São Paulo: Editora Atheneu, 1996. Vol. 5, p. 43-100. 
37. Tomasz, A.; Albino, A. \& Zanati, E. Multiple antibiotic resistance in a bacterium with suppressed autolytic system. Nature, 227: 138-40, 1970.

38. Voorn, G.P. et al. Role of tolerance in treatment and prophylaxis of experimental Staphylococcus aureus endocarditis with vancomycin, teicoplanin, and daptomycin. Antimicrob. Agents Chemother., 38: 487-93, 1994.

39. Voorn, G.P. et al. In vitro development and stability of tolerance to cloxacillin and vancomycin in Staphylococcus aureus. Eur. J. Clin. Microbiol. Infect. Dis., 13: 741-6, 1994.
40. Watanakunakorn, C. Antibiotic tolerance of Staphylococcus epidermidis. Scand. J. Infect. Dis., 17: 59-61, 1985.

41. Yao, J.D.C. \& Moellering, R.C. Antimicrobial agents. In: Murray, P.R. et al. Manual of clinical microbiology. 7.ed. Washington: American Society for Microbiology, 1991. Vol. 116, p. 1474-504.

42. Zaoutis, T. et al. Antibiotics susceptibilities of group C and $G$ Streptococci isolated from patients with invasive infections: evidence of vancomycin tolerance among group $\mathrm{G}$ serotypes. J. Clin. Microbiol., 37: 3380-3, 1999. 